\title{
Position Sensing System for Switched Reluctance Motor Control
}

\author{
Rareș Terec*, Virgil Chindriş*, Loránd Szabó*, Pavol Rafajdus ${ }^{\dagger}$ \\ * Technical University of Cluj-Napoca, Department of Electrical Machines and Drives \\ Cluj-Napoca, Romania, e-mail: Lorand.Szabo@mae.utcluj.ro \\ ${ }^{\dagger}$ University of Žilina, Department of Power Electrical Systems \\ Žilina, Slovak Republic, e-mail: pavol.rafajdus@kves.uniza.sk
}

\begin{abstract}
Switched reluctance motors driven with modern power electronics and using electronic controls are more and more often considered as a feasible alternative to conventional motors in diverse variable speed applications. Their simple construction, high reliability and efficiency make them suitable both for industrial and automotive applications. A modern, PIC and dsPIC Microchip microcontroller board based implementation of their control is discussed. The focus is on the position sensing.
\end{abstract}

Keywords-Switched reluctance motor, control, position sensing, incremental encoder, PIC and dsPIC microcontroller boards.

\section{INTRODUCTION}

The switched reluctance motors (SRM) are some of the most significant new developments in the field of electrical machines for variable speed drives. They have several attractive features, such as rugged and robust construction and low manufacturing costs, high starting torque, wide speed range, etc. [1], [2]. Being a variable reluctance type machine it must be obligatory controlled electronically.

To achieve good performances the SRM requires an accurate shaft position feedback signal, implying the need for a good quality encoder or, alternatively, a sophisticated sensorless controller [3], [4], [5].

The position sensor used in SRM drives have the disadvantages of additional cost, electrical connections, mechanical alignment problems, less suitability to space restricted applications and significant disadvantage of being inherent source of unreliability.

On the other hand the sensorless controllers need consistent extra computation time and memory to cover the huge amounts of mathematical operations and large lookup tables required by the implementation of the advanced control strategy. Therefore they need costly very high speed processors. Due to these drawbacks most of the commercial SRM controllers use positions sensors.

The control strategy was implemented on coupled PIC and dsPIC Microchip microcontroller boards. Via the Delphi software platform a graphic user interface (GUI) has been created [6]. By using the mikroPascal language both the PIC and the dsPIC boards have been specifically programmed for their proper purpose. The PIC board serves as a connection for the PC to the dsPIC board, and the dsPIC board provides the necessary command tools and functions.
In the paper the implemented control system is presented, focusing mainly on the rotor's position sensing.

\section{The Switched Reluctance Motor}

The SRM is a double salient electrical machine with a passive rotor [7]. The torque is produced by the tendency of its rotor to get to a position where the inductance and the flux produced by the energized stator winding are maximized (variable reluctance principle) [8].

The SRM's rotor and stator both have salient poles, as shown in Fig. 1.

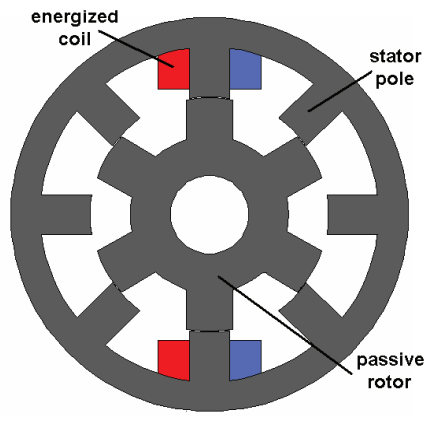

Fig. 1. The cross section of a four phase $8 / 6 \mathrm{SRM}$.

The stator is formed from punched laminations that have been bonded into a stack. The rotor is also made of conventional laminations without any kind of winding, excitation, squirrel-cage or permanent magnets [9].

The windings consist of coils placed on the stator poles. Each independent phase is fed in turn thru a half H-bridge. By opening and closing the transistors the correct unipolar current sequence required by the SRM is assured. Therefore the SRM cannot be fed from convention one or three phase source and then cannot be separated from the electronic supply device and its control [7].

The phase excitation pulses of the SRM need to be properly synchronized with the rotor position for effective control of speed, torque and torque pulsations, hence the precise rotor position information is essential for the control system [1], [10].

The various advantages of the SRM make it an attractive alternative to the existing dc and ac motors in adjustable speed drives. The SRM drives can also deliver servo performances. The rotor position sensing requirements, the need for an electronic converter and the higher noise and torque ripple, compared to other machines, are the main disadvantages of the SRM drives. 
The most common control system of the SRM is given in Fig. 2.

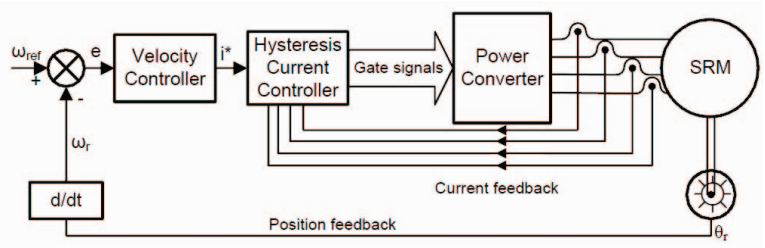

Fig. 2. The SRM's control system

The machine is equipped with a position sensor. The angular position is red by this sensor and the signal is sent to the controller. Deriving the position in the time domain the angular speed of the rotor can be computed. The controller compares the actual speed with the reference value. Upon the error signal the current reference for the hysteresis comparator is calculated. Each phase is supplied within a certain rotor position range in order to maximize the developed torque. The hysteresis controllers are sending the gate signals to the power switches of the converter.

\section{ThE PROPOSED SRM CONTROLLER}

The block scheme of the proposed controller is given in Fig. 3.

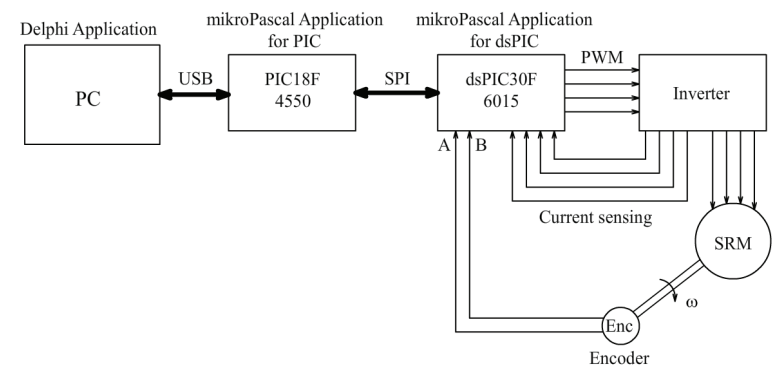

Fig. 3. The block scheme of the proposed SRM controller.

The proposed system uses four devices to control the motor and an incremental encoder to determine the rotor's position [11]. These devices are: a PC, a PIC based board, a dsPIC board and a four-phase inverter. The controller unit requires the intermediary PIC based board to link the $\mathrm{PC}$ to the primary command module, the dsPIC board.

The PIC board (in the right of Fig. 4) features a series of connectors that provide SPI (Serial Peripheral Bus) functionality. Through this bus the two boards communicate in master/slave mode, where the data transmission is initialized by the master [12], [13].

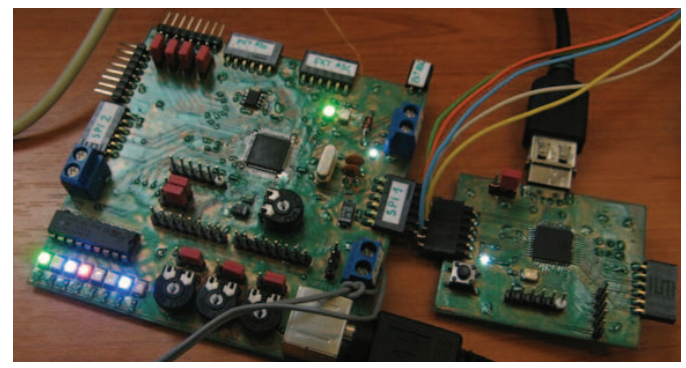

Fig. 4. The dsPIC and PIC board.

The PIC board has a PIC18F4550 microcontroller as the main processing unit with a USB connection to the PC for high speed access. It also contains a power supply jumper, a programmer connector and some LEDs that indicate the on/off state of the board, and of the USB connection. There is a jumper that enables the board to be powered by either the USB connection or from the dsPIC. It is connected to the dsPIC board via connector pins.

The dsPIC board (in the left of Fig. 4) has a dsPIC 30F6015 microcontroller as the main processing unit. It has a larger number of connectors and components. There are 3 jumpers (for the $A, B$ and index signals of the encoder) that enable the dsPIC to interface with more than one type of encoder, using variable resistors to protect the dsPIC from the $12 \mathrm{~V}$ voltage used by the encoder. A PS/2 connector is used to interface with the encoder.

Several other units are at the user's disposal on the board: a programming connector, 4 PWM generators (each having the outputs connected to 2 pins, so that there are 8 pins used for the command), a power supply connector, an oscillator that generates the clock signal for the dsPIC, several user defined components, such as LEDs and connectors, etc. The PWM generators are linked to 8 LEDs, showing the on/off at a certain moment [14]. They also provide minimal information in terms of debugging, the intensity being linked to the fill-factor.

The dsPIC board is the main module of the SRMs's controller. It is supplying all the basic functions and, when it is necessary, the modified command in order to overcome faults and maintain the functionality for a given time period [15].

By using Delphi a simple and easy-to-use (GUI) was developed for setting the main parameters of the control system and for visualization purposes.

Using artificial intelligence (AI) implementation the efficiency of the system can be increased even further [16]. Such an approach would be useful when attempting to find an optimized control pattern for the command angles, with a program on the PC.

The dsPIC's PWM generators are configured by the program. During a typical working regime the dsPIC sends the PWM command signals to the inverter. The duty cycle is adjusted according to the operation requirements [17]. The motor's coils being energized sequentially the rotor revolves at the required speed. The encoder sends out information about the position of the rotor through impulses.

\section{The Position SEnsing}

In order to correctly select which phase of the machine has to be energized the precise position of the machine is required.

For this purpose the proposed SRM controller uses a Siemens 1XP8001-1 (1024) type incremental encoder mounted on the shaft of the SRM, as shown in Fig. 5.

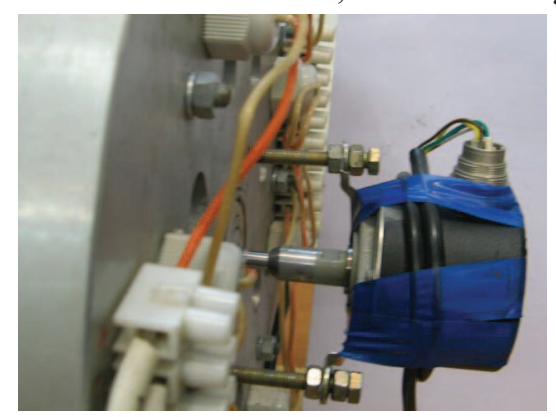

Fig. 5. The encoder mounted on the shaft of the SRM. 
The signals obtained from the position sensor have to be electronically processed [18].

The encoder delivers two signals, $A$ and $B$, which provide information about the sense of rotation, and indirectly, about the speed.

The waveforms of the signals from the encoder are given in Fig. 6.

Clockwise Rotation
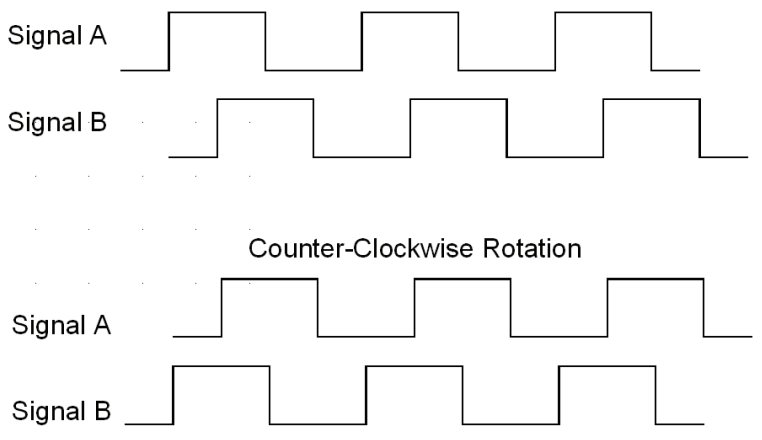

Fig. 6. Signals $A$ and $B$ from the encoder.

The encoder delivers 1024 impulses/revolution. Hence with the given decoder (a device which performs the reverse operation of an encoder by retrieving the information previously encoded), and based by the signals in a quadrature, $1024 \times 4=4096$ transitions per revolution can be obtained (see Fig. 7).

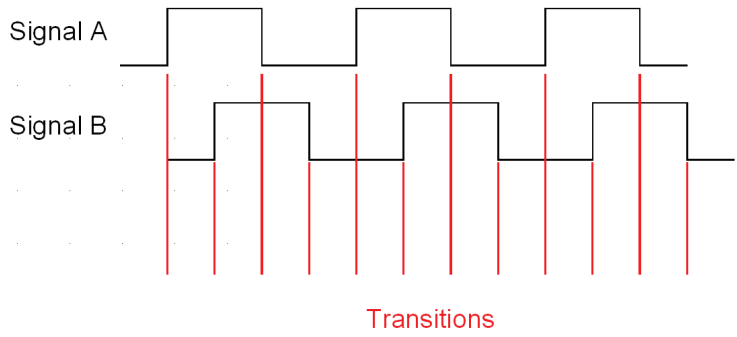

Fig. 7. Transitions of the encoder signals (the red lines).

Basically, for a $60 \mathrm{r} / \mathrm{min}$ speed, there is one revolution per second, which means 4096 transitions per second. At this speed by using a frequency meter $4096 \mathrm{~Hz}$ would be measured.

From these impulses the speed can be computed. It can also be determined by using an interrupt sequence with a timer, and at each interrupt, the position of the encoder can be calculated from the difference between that current and a previous value.

The circuit schematic for detecting the sense of rotation is given in Fig. 8 .

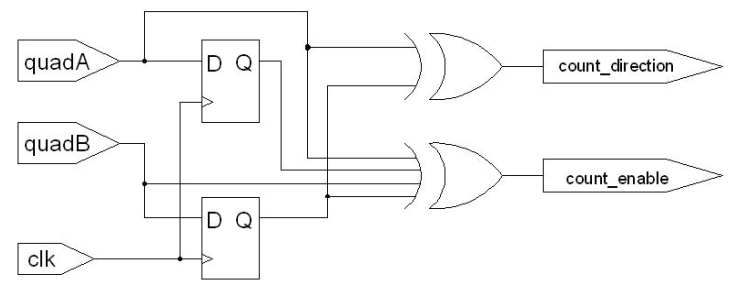

Fig. 8. Direction detection circuit schematic.

The inputs quad $A$ and quadB (the signals in quadrature) are connected to the $A$ and respectively $B$ signals of the encoder. Two D-type flip-flop gates sample the signals and at each transition the clock (clk), the output of gate, $Q$, copies the value of the input, $D$. Therefore, the input $D$ features the current value; respectively the output $Q$ features the previous one.

In order to use these signals, a decoder is required. The dsPIC has an option for using an on-chip peripheral for such purpose. The working principle is simple: the decoder determines the number of impulses or the number of transitions of the $A$ and $B$ signals and allows a counter to keep a record of them.

Count direction uses a XOR gate to obtain the direction of the rotation (a XOR gate returns '1' when the number of ' 1 ' inputs are odd numbers (1,3 etc). Therefore, by taking the 4 signals as inputs, each time there is an odd number of '1', meaning when there is a transition of the input signals

The vertical lines are the moment of sampling. Taking into account the last ' 0 ' value, the flip-flop gate copies that value from the $D$ entrance to the $Q$ exit, while at the next sampling, the value will be changed to ' 1 '. So the input and output of the signals are different. Through the 4 inputs it can be determined whether the encoder moved or not.

The XOR gate with 4 inputs from Fig. 8 allows the counter to increment or decrement (based on the sense of rotation determined by the XOR gate with 2 inputs) the impulse number at each transition.

A basic problem in the relative position sensing is finding of a reference position. Before starting the SRM a specific phase of the motor is energized, which will force the rotor to move to its reference position. When this is achieved the encoder's counter is reset to 0 .

Having the actual position of the SRM determined also the average speed can be computed from at least 3 (depending on the accuracy required) time delays measured between 3 known angular positions.

\section{MEASUREMENTS}

A test bench was built up to test the position sensing function of the proposed control system. It is composed of a four-phase SRM of modular stator construction [18], the control system described in Section III, and a dc voltage source for feeding the encoder (see Fig. 9).

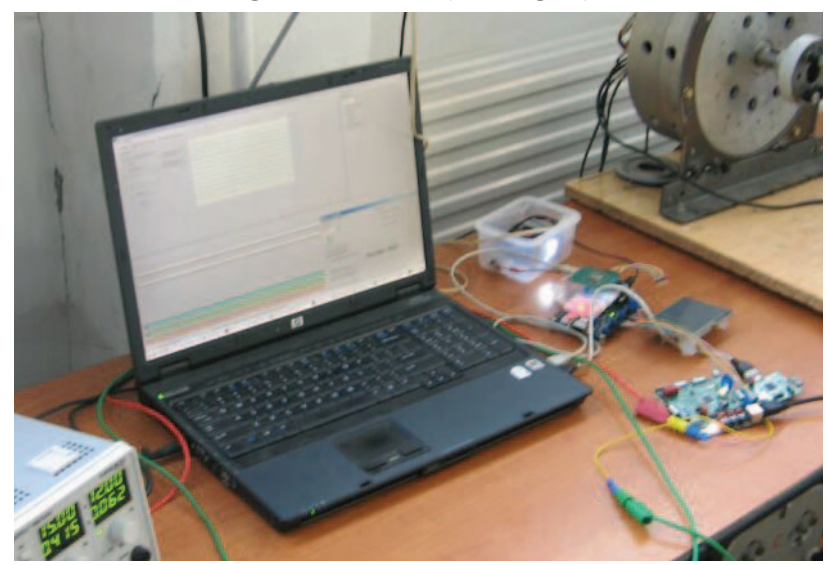

Fig. 9. The test bench.

In Fig.10 three sets of pulse train waveforms of the communications between the dsPIC and PIC are given. The sets of waveforms acquired at different moments. The $C S$ (chip select) signal enables the slave device. The SDI 

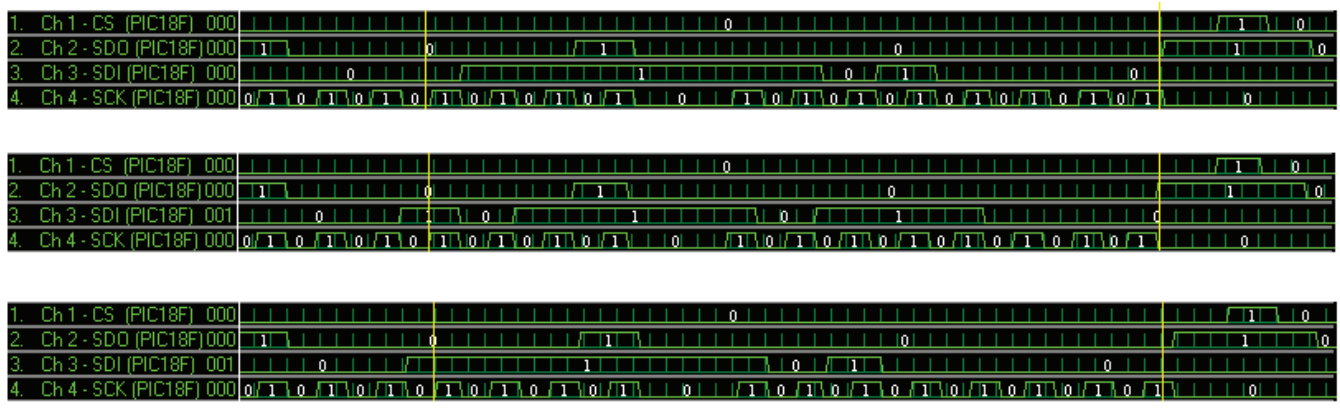

Fig. 10. The pulse train waveforms of the communications between the dsPIC and PIC.

and SDO signals are basically the command output and input, the second one being the binary representation of the current impulse's number. The $S C K$ (clock signal) synchronizes the master with the slave. Data transfer is performed at each program defined transition of the clock. In this case on the positive transition (meaning from ' 0 ' to '1') a bit is being transferred.

The first capture shows the $2000^{\text {th }}$ impulse, the second one the $3000^{\text {th, }}$ was taken $0.24 \mathrm{~s}$ later, and the last one is the $4000^{\text {th }}$ impulse, generated after $0.48 \mathrm{~s}$ from the first sample. The encoder pane of the controller's GUI for the third case is given in Fig 11. The detected angular positions are: $3.06 \mathrm{rad}, 4.6 \mathrm{rad}$, respectively $6.13 \mathrm{rad}$.

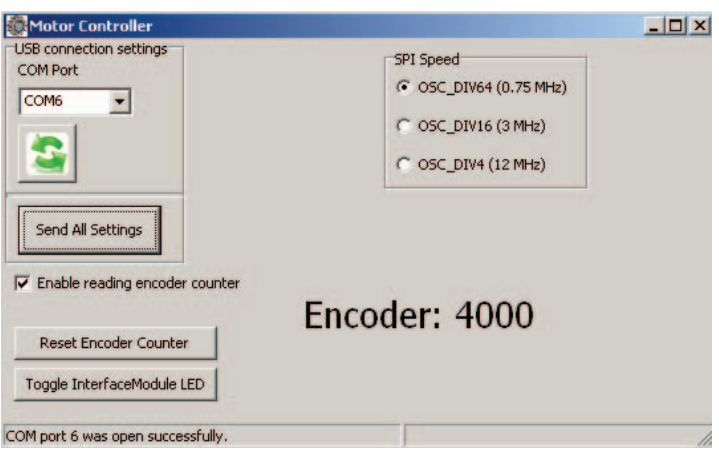

Fig. 11. The Motor Controller window.

Therefore, the speed of the SRM is roughly $60 \mathrm{r} / \mathrm{min}$ (1 revolution per second), the speed of the rotor as it was revolved from an external motor.

\section{CONCLUSIONS}

As the commutation of the SRM's phase currents must be synchronized precisely with the rotor position it is very important to have at any given time moment precise information on the SRM's angular position. The control system proposed in this paper (implemented on a dsPIC and a PIC board) can perform perfectly this exigent task.

\section{ACKNOWLEDGMENT}

This paper was supported by the project "Doctoral studies in engineering sciences for developing the knowledge based society - SIDOC" contract no. POSDRU/88/1.5/S/60078, project co-funded from European Social Fund through Sectorial Operational Program Human Resources 2007-2013, respectively by the research grant "Improved performance variable reluctance machines for safety-critical industrial process applications" no. SK-RO-0016-10 in the framework of the "Romanian-Slovak Intergovernmental S\&T Cooperation Programme for 2011-2012".

\section{REFERENCES}

[1] T.J.E. Miller, Electronic Control of Switched Reluctance Machines. Oxford (U.K.): Newnes, 2001.

[2] G. Baoming, et al., "Nonlinear internal-model control for switched reluctance drive with torque ripple-free," Automatika, vol. 43, pp. 2-13, 2002.

[3] T. Miller, "Optimal design of switched reluctance motors," IEEE Transactions on Industrial Electronics, vol. 49, pp. 15-27, 2002.

[4] M. Ehsani and B. Fahimi, "Elimination of position sensors in switched reluctance motor drives: state of the art and future trends," IEEE Transactions on Industrial Electronics, vol. 49, pp. 40-47, 2002.

[5] C. Kamalakannan, et al., "Switched reluctance machine in automotive applications - A technology status review," in Proc. of the 1st Intern. Conf. on Electrical Energy Systems (ICEES '2011), Newport Beach (CA, USA), 2011, pp. 187-197.

[6] M. Cantù, Mastering Borland Delphi 2005. Indianapolis (USA): SYBEX, 2006.

[7] G. Henneberger and I. A. Viorel, Variable Reluctance Electrical Machines. Aachen (Germany): Shaker Verlag, 2001.

[8] S. Masic, et al., "Computation of static, steady-state and dynamic characteristics of the switched reluctance motor," Automatika, vol. 43, pp. 109-117, 2002.

[9] R. Krishnan, Switched reluctance motor drives: modeling, simulation, analysis, design, and applications. Boca Raton (USA): CRC, 2001.

[10] I. Husain and M. Ehsani, "Rotor position sensing in switched reluctance motor drives by measuring mutually induced voltages," IEEE Transactions on Industry Applications, vol. 30, pp. 665-672, 1994.

[11] A. Fehn and R. Rothfuß, "Control of switched reluctance servodrives," Control and Observer Design for Nonlinear Finite and Infinite Dimensional Systems, vol. 322, pp. 301-313, 2005.

[12] D. Ibrahim, "Serial Peripheral Interface Bus Operation," in SD Card Projects Using the PIC Microcontroller, ed Burlington (MA, USA): Newnes, 2010.

[13] D. Ruimy Gonzales, "Serial peripheral interfacing techniques," Microelectronics, vol. 17, pp. 5-14, 1986.

[14] G.C. Goodwin, et al., "An introduction to the control of switching electronic systems," Annual Reviews in Control, vol. 34, pp. 209$220,2010$.

[15] R. Terec, et al., "Fault detection in switched reluctance machines," Journal of Computer Science and Control Systems, vol. 3, pp. 231236, 2010.

[16] V. Uraikul, et al., "Artificial intelligence for monitoring and supervisory control of process systems," Engineering Applications of Artificial Intelligence, vol. 20, pp. 115-131, 2007.

[17] H. Hannoun, et al., "High performance current control of a switched reluctance machine based on a gain-scheduling PI controller," Control Engineering Practice, vol. 19, pp. 1377-1386, 2011.

[18] M. Ruba, "Design and Study of a Modular Switched Reluctance Machine," Ph.D., Electrical Machines Department, Technical University of Cluj, Cluj (Romania), 2010. 